\title{
ORGANIC SYNTHESIS IN THE LATE STAGES OF STELLAR EVOLUTION
}

\author{
S. Kwok ${ }^{1}$
}

\begin{abstract}
Infrared and mm-wave observations have revealed a rapid and continuous synthesis of gas-phase and solid organic compounds in the late stages of stellar evolution. This process gives rise to an amorphous carbonaceous compound of mixed aromatic and aliphatic structure which is probably responsible for the unidentified infrared emission (UIE) bands that emerge during the PN phase.
\end{abstract}

\section{Introduction}

Mass loss from asymptotic giant branch (AGB) stars was discovered as the result of advances in infrared and $\mathrm{mm}$-wave technologies. The observations of infrared excess due to circmstellar dust emission and the detection of molecular-line emission of $\mathrm{CO}$ and $\mathrm{OH}$ in evolved stars were recognized as manifestation of mass loss (Kwok 1987). The rotational and vibrational transitions of over 80 gas-phase molecules (including many organics) have been detected in the stellar wind outflow from AGB stars. Solid-state minerals (amorphous silicates, silicon carbide, and refractory oxides) are commonly detected in the circumstellar envelopes of AGB stars, and these solid particles are believed to have condensed from gas-phase molecules in the stellar outflow (Kwok et al. 1997).

The most surprising discovery was the detection of a family of UIE bands in carbon-rich planetary nebulae (PN), the descendents of AGB stars. The lifetimes of PN are of the order of $10^{4} \mathrm{yr}$ and the transition from AGB to PN through the proto-planetary nebulae (PPN) phase only takes a few thousand years. If the carrier of the UIE bands are carbon-based molecules or solids, then carbon-based chemical synthesis must be actively taking place in the circumstellar environment.

\section{The unidentified infrared emission bands phenomenon}

The UIE bands phenomenon has the following properties: (1) aromatic features at $3.3,6.2,7.7,8.6$, and $11.3 \mu \mathrm{m}$; (2) aliphatic features at 3.4 and $6.9 \mu \mathrm{m}$;

\footnotetext{
${ }^{1}$ Faculty of Science, The University of Hong Kong, Hong Kong, China
} 
(3) unidentified features at 15.8, 16.4, 17.4, 17.8, and $18.9 \mu \mathrm{m}$ (in PPN, Kwok et al. 1999, in reflection nebulae; Sellgren 2007, in galaxies; Sturm et al. 2000); and

(4) broad plateau features at 8, 12, and $17 \mu \mathrm{m}$ (Kwok et al. 2001).

In the past 25 years, the most popular model for the UIE phenomenon is the polycyclic aromatic hydrocarbon $(\mathrm{PAH})$ hypothesis. The $\mathrm{PAH}$ model assumes that the UIE features are the result of infrared fluorescence from small ( $\sim 50 \mathrm{C}$ atoms) gas-phase PAH molecules being pumped by far-ultraviolet photons (Tielens 2008). The central argument for the PAH hypothesis is that single-photon excitation of PAH molecules can account for the $12 \mu \mathrm{m}$ excess emission observed in cirrus clouds in the diffuse interstellar medium by IRAS (Sellgren 2001).

However, the PAH hypothesis suffers from a number of problems: (1) PAH molecules have well-defined sharp features but the UIE features are broad; (2) PAH molecules are primarily excited by UV with weak absorption in the visible, but UIE features are seen in PPN and reflection nebulae with little UV radiation; (3) the strong electronic absorption transitions of PAH molecules in the UV are not seen along the lines of sight to strong UV sources, implying upper limits of $n_{\mathrm{PAH}} / n_{\mathrm{H}}=10^{-10}-10^{-8}$ (Salama et al. 2011; Gredel et al. 2011), which are much lower than the abundances predicted from the strengths of the infrared emission features; (4) no specific PAH molecules have been detected in spite of the fact that the vibrational and rotational frequencies of PAH molecules are well known; (5) the shapes and peak wavelengths of UIE features are independent of temperature of exciting stars; and (6) no laboratory PAH emission spectrum has been able to reproduce the UIE spectrum w.r.t. either band positions or relative intensities (Cook et al. 1998; Wagner et al. 2000).

In order to fit the astronomical observations, the PAH model has to appeal to a mixture of PAH of different sizes, structures (compact, linear, branched) and ionization states, as well as artificially broaden intrinsic line profiles. The NASA Ames PAH database contains 700 computational and 75 experimental spectra of $\mathrm{PAH}$ molecules and ions with size range from 6 to $384 \mathrm{C}$ atoms, neutral, anion, and cations charged states and this database is used to fit the astronomical UIE bands (Boersma et al. 2014). However, the fitting routines are so flexible that the PAH model can also fit astronomical spectra of amorphous silicates, laboratory spectra of coal and hydrogenated amorphous carbon, and even artificially generated random spectra (Zhang \& Kwok 2015).

\section{The MAON model}

By introducing $\mathrm{H}$ into graphite $\left(s p^{2}\right)$ and diamond $\left(s p^{3}\right)$, a variety of amorphous $\mathrm{C}-\mathrm{H}$ alloys can be created. Geometric structures of different long- and short-range can be created by varying the aromatic to aliphatic ratio. Examples of such amorphous hydrocarbons are soot (a natural product of combustion) or coal (Papoular et al. 1989). Figure 1 shows a comparison between the laboratory spectrum of artificially created carbonaceous nanoparticles to the astronomical spectrum of the PN IRAS 21282+5050. There are obvious qualitative similarities between these spectra. 

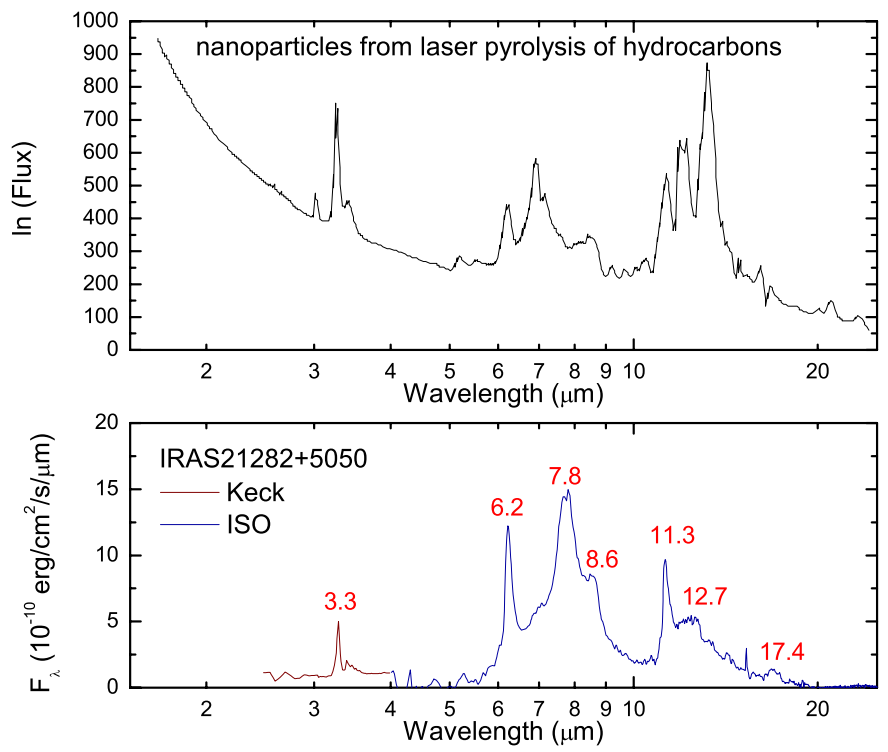

Fig. 1. Comparison of the laboratory spectrum of nanoparticles produced by laser pyrolysis of hydrocarbons (Herlin et al. 1998) (top panel) with the astronomical spectrum of the PN IRAS 21282+5050 (bottom panel). Figure taken from Sadjadi et al. (2015).

As an alternative to the PAH hypothesis, a mixed aromatic/aliphatic organic nanoparticles (MAON) model is suggested to be the carrier of the UIE bands (Kwok \& Zhang 2011, 2013). The MAON model is different from the PAH model in several ways: (1) MAON are amorphous and have no fixed structure; (2) MAON contain rings of different sizes and chains of different lengths and random orientations; (3) MAON are not pure hydrocarbons but contain impurities; (4) they are $3-\mathrm{D}$, not 2-D as in the case of PAH; and (5) the exact aromatic to aliphatic ratio depends on radiation background, the mix of gas-phase ingredients, and $\mathrm{H}$ content in the environment.

Although the formation of substances as complex as MAON in the interstellar environment may seem difficult theoretically, yet we can observe aliphatic and aromatic compounds forming in PPN on time scales as short as hundreds of years. In novae, the formation time scale is even shorter, in a matter of weeks or days. Nature therefore has no problem forming complex organics even in a very low density environment.

\section{Organics in the early universe}

The UIE bands have been detected in $\mathrm{PN}$, reflection nebulae, $\mathrm{H}$ II regions, diffuse interstellar medium, and external galaxies. In some active galaxies, up to $20 \%$ of the total luminosity of the galaxy is emitted in the UIE bands (Smith et al. 2007). The detection of UIE bands in high-redshift galaxies (Teplitz et al. 2007) implies 
that complex organics were widely present as early as 10 billion years ago. This suggests that abiological synthesis of complex organics has been occurring through most of the history of the Universe. Since the UIE bands are commonly used to trace star formation and to probe the radiation field of galaxies assuming that PAH molecules are the carrier (Valiante et al. 2007; Pope et al. 2013), a correct identification of the UIE bands are essential for our understanding of the galactic environment.

I would like to thank Seyed Abdolreza Sadjadi and Yong Zhang for helpful discussions. This work was partially supported by the Research Grants Council of the Hong Kong Special Administrative Region, China (project No. 17302214).

\section{References}

Boersma, C., et al., 2014, ApJS, 211, 8

Cook, D.J., et al., 1998, J. Phys. Chem. A, 102, 1465

Gredel, R., et al., 2011, A\&A, 530, 26

Herlin, N., et al., 1998, A\&A, 330, 1127

Kwok, S., 1987, Physics Reports, 156, 111

Kwok, S., \& Zhang, Y., 2011, Nature, 479, 80

Kwok, S., \& Zhang, Y., 2013, ApJ, 771, 5

Kwok, S., Volk, K., \& Bidelman, W.P., 1997, ApJS, 112, 557

Kwok, S., Volk, K., \& Hrivnak, B.J., 1999, A\&A, 350, L35

Kwok, S., Volk, K., \& Bernath, P., 2001, ApJ, 554, L87

Papoular, R., Conrad, J., Giuliano, M., et al., 1989, A\&A, 217, 204

Pope, A., et al., 2013, ApJ, 772, 92

Sadjadi, S., Zhang, Y., \& Kwok, S., 2015, ApJ, 807, 95

Salama, F., Galazutdinov, G.A., Krelowski, J., et al., 2011, ApJ, 728, 154

Sellgren, K., 2001, Spectrochimica Acta, 57, 627

Sellgren, K., Uchida, K.I., \& Werner, M.W., 2007, ApJ, 659, 1338

Smith, J.D.T., et al., 2007, ApJ, 656, 770

Sturm, E., et al., 2000, A\&A, 358, 481

Teplitz, H.I., et al., 2007, ApJ, 659, 941

Tielens, A.G.G.M., 2008, ARA\&A, 46, 289

Valiante, E., et al., 2007, ApJ, 660, 1060

Wagner, D.R., Kim, H., \& Saykally, R.J., 2000, ApJ, 545, 854

Zhang, Y., \& Kwok, S., 2015, ApJ, 798, 37

\section{Discussion}

L. Guzman: is it possible to fit the Fullerene features and relate them to the MAON?

S. Kwok: after fullerene was discovered in planetary nebulae and protoplanetary nebulae, it was found that $\mathrm{C}_{60}$ sources also show 8 and $12 \mu \mathrm{m}$ plateaus. It has been suggested that $\mathrm{C}_{60}$ can be precursors of the carrier of UIE bands. 
A. Moffat: one may not have to wait for intermediate stars to take 100s of millions of years to evolve to AGBs to produce carbon and organics. The first stars to form in the Universe will have burnt the to produce carbon after only a few million years. S. Kwok: massive stars are the first to evolve. However so far we have only seen UIE bands in planetary nebulae and proto-planetary nebulae, but not in ejecta of massive stars. 
Copyright of EAS Publications Series is the property of EDP Sciences and its content may not be copied or emailed to multiple sites or posted to a listserv without the copyright holder's express written permission. However, users may print, download, or email articles for individual use. 